\title{
HEH-BMAC: Hybrid Polling MAC Protocol for WBANs Operated by Human Energy Harvesting.
}

\author{
Ernesto Ibarra • Angelos Antonopoulos • Elli Kartsakli • \\ Christos Verikoukis
}

Received: date / Accepted: date

\begin{abstract}
This paper introduces Human Energy Harvesting Medium Access Control (MAC) protocol (HEHBMAC), a hybrid polling MAC suitable for Wireless Body Area Networks (WBANs) powered by human energy harvesting. The proposed protocol combines two different medium access methods, namely polling (IDpolling) and probabilistic contention (PC) access, to adapt its operation to the different energy and state (active/inactive) changes that the network nodes may experience due to their random nature and the time variation of the energy harvesting sources. HEH-BMAC exploits the packet inter-arrival time and the energy harvesting rate information of each node to implement an efficient access scheme with different priority levels. In addition, our protocol can be applied dynamically in realistic networks, since it is adaptive to the topology changes, allowing the insertion/removal of wireless sensor nodes. Extensive simulations have been conducted in order to evaluate the protocol performance and study the throughput and energy tradeoffs.
\end{abstract}

Keywords Energy efficiency, WBAN, energy harvesting, MAC.

Ernesto Ibarra $₫$, Angelos Antonopoulos and Christos Verikoukis are with the Telecommunications Technological Centre of Catalonia (CTTC), Castelldefels, Barcelona, Spain.

Elli Kartsakli is with the Signal Theory and Communications Department, Universitat Politecnica de Catalunya (UPC), Barcelona, Spain.

Corresponding author. Tel.: +34 936452900 .

E-mail addresses: eibarra@cttc.es (E. Ibarra), aantonopoulos@cttc.es (A. Antonopoulos), ellik@tsc.upc.edu (E. Kartsakli), cveri@cttc.es (C. Verikoukis).

\section{Introduction}

Wireless Body Area Networks (WBANs) are specified in the IEEE 802.15.6 Standard for short-range wireless communications in the vicinity of, or inside, a human body 41]. A WBAN consists of small, intelligent devices, also called body sensor nodes, with sensing, processing and wireless communication capabilities, able to act without assistance from other devices. The nodes that form part of the WBAN are devices capable of performing one or more actions with respect to the monitoring of physiological parameters, diagnosis and treatment of diseases.

WBANs differ from typical Wireless Sensor Networks (WSNs) in terms of technical requirements, components, size and application scenarios [3] [16 [26. A WBAN is usually composed of few body sensor nodes, even though up to 256 nodes are supported by the IEEE 802.15.6 standard. A relatively wide range of data rates can be employed, varying from $1 \mathrm{kbps}$ to $100 \mathrm{Mbps}$. The wireless body sensors are devices that inherently face certain restrictions and problems due to their interaction with the human body and the functions they perform on it. In particular, the power available in the nodes is often restricted. A battery-powered wireless node can maintain its functions efficiently (detection, processing, reception, transmission, etc.) provided that the battery level is sufficient for the node to operate. The power consumption is a critical metric that defines the scope, usefulness and lifetime of the network [15.

There have been several works in the literature that aim to increase the lifetime of the network powered by batteries [4 21] 27. However, as energy is consumed, the node may be forced to reduce its activities 


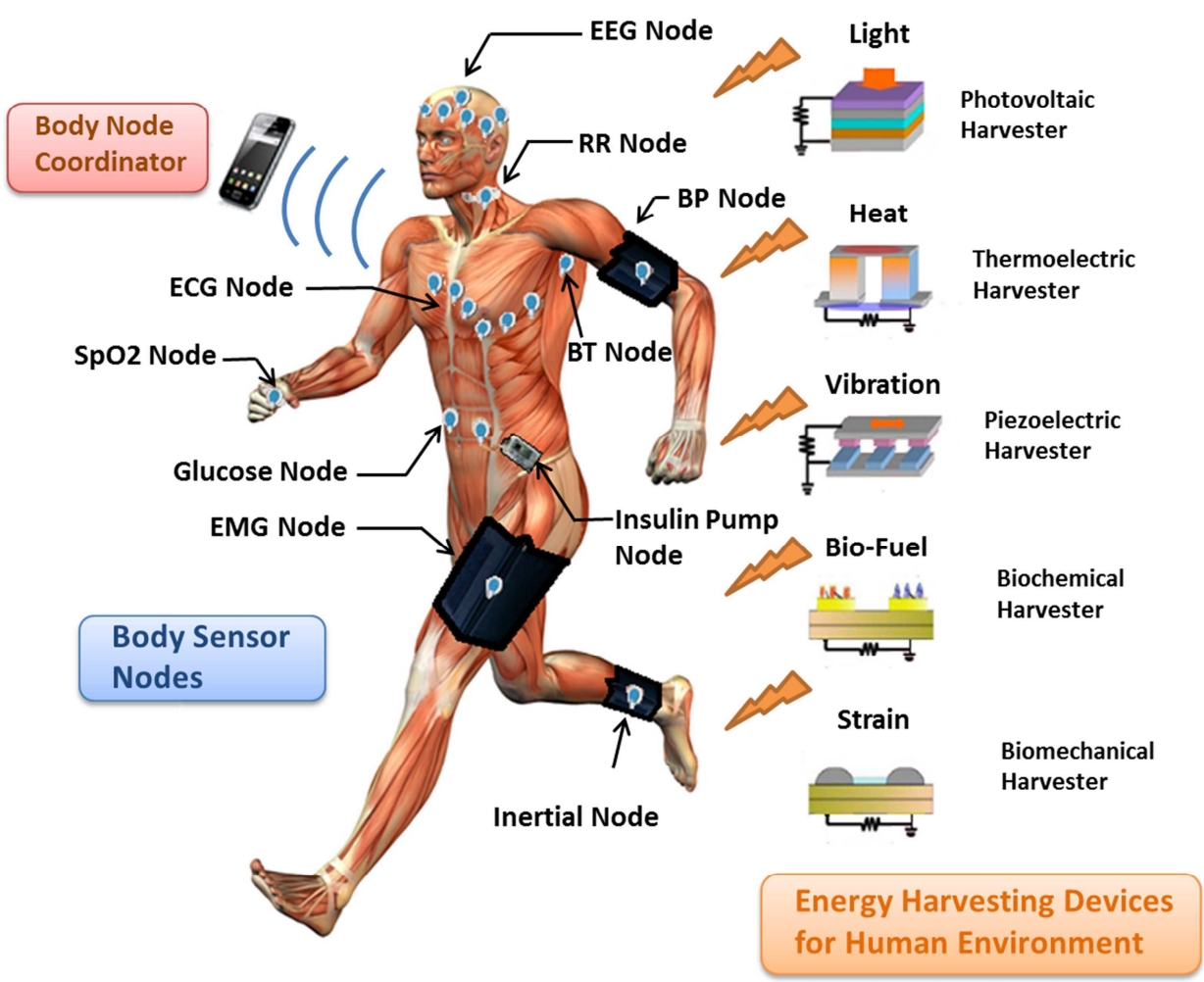

Fig. 1 Wireless body area network operated by human energy harvesting (e.g. Electroencephalogram (EEG), respiratory rate $(\mathrm{RR})$, blood pressure (BP), body temperature (BT), electromyogram (EMG), blood oxygen saturation SpO2, and electrocardiogram (ECG)).

before becoming permanently inactive when its power is totally depleted. One method to prolong the lifetime of the nodes is to increase the energy capacity of the battery. Unfortunately, the battery capacity is proportional to its size and weight. Wearable sensors are often small in size, thus posing restrictions to the battery size as well. Since the battery replacement is not easy and usually requires a surgical procedure, the case of implanted sensors is even more complicated.

To overcome the above limitations, an infinite source of energy harvesting or energy scavenging that could power the node permanently constitutes the most promising and encouraging solution for this problem [23] [7. The harvested energy should be ideally collected and stored in a rechargeable device, which could be a battery or a super-capacitor. The super-capacitor is the most suitable element for this work because of its almost infinite recharging cycles 32, 36. Energy harvesting can significantly extend the functionality of a wireless body sensor node; however, there are complex tradeoffs to be considered in the design of energy harvesters 34. These tradeoffs are related to the interaction of several factors, such as the characteristics of the energy sources, the energy storage devices, the power management of the nodes, the applied protocols and the application requirements. In addition, energy harvesters specifically for WBANs differ from those used in traditional WSNs. Currently, solar, mechanical, thermal and electromagnetic energy are the main sources of ambient energy to be applied in WSNs [9] 10. On the other hand, the power supply of body sensor nodes could be accomplished by harvesting energy from multiple sources within the human body 5 , including mechanical [13] [1, thermal 24] 28, and biochemical 20 31 energy to enhance or even replace traditional batteries. In this case, energy harvesting will only deliver small amounts of energy compared to the ambient sources, while it is more time dependent. Figure 1 illustrates an example of a WBAN employing human energy harvesting. The Figure 1 depicts several types of body sensor nodes and energy harvesters. The harvesters use different phenomena to produce energy, such as light (photovoltaic harvester), vibration (piezoelectric harvester), strain (biomechanical harvester), heat (thermoelectric harvester) and bio-fuels (biochemical harvester).

In order to exploit the additional energy provided to the WBAN through energy harvesting, it is important to design energy efficient mechanisms. The MAC sub- 
layer, part of the data link layer, is the most appropriate level to address energy efficiency, since it performs functions related to packet transmission, flow control, data rate selection and energy management. The authors in 22 provide a comparative analysis of the energy efficiency of MAC protocols in the context of WBANs, such as: S-MAC [42, T-MAC [39], B-MAC [30], DQBAN [6], MedMAC [38, Energy-Efficiency Low Duty Cycle MAC Protocol [29], and BodyMAC [19, among others. Currently, most MAC protocols for WBANs are specially designed to optimize energy consumption by implementing actions related to the battery management. However, the effects introduced by power sources based on energy harvesting are not effectively covered by existing schemes.

In the literature, some works model and analyze the energy harvesting effect in WBANs using probabilistic models based on Markov chains, making optimal numerical solutions for energy efficient transmission strategies and resource allocation [2] 8] 35] [40. In none of the cited works, though, a MAC protocol that supports energy harvesting techniques is proposed. Eu et al. 18] show the design, performance and comparative analysis of MAC schemes for WSNs powered by ambient energy harvesting. In [17, Eu and Tan proposed EH-MAC, a MAC protocol for multi-hop energy harvesting WSNs. EH-MAC is a MAC protocol based on probabilistic contention that adapts its operation to the energy harvesting rates and/or the number of nodes to achieve high throughput, fairness and scalability.

In this point, we should note that the clinical environment is extremely changeable. The importance of each node depends on the current health conditions of the patient and studied parameters at any given time. For this reason, adding and removing nodes in a fast and easy way is a very desirable feature in WBANs. Unfortunately, the application of EH-MAC in WBANs operated by energy harvesting is not straightforward, since the access for all nodes is probabilistic, thus not assigning different level of priorities to the nodes. In WBANs, the early detection of crucial events is of vital importance, since it provides information about the patient's health. Although data loss in typical WSNs can be compensated by the number of sensor nodes, this is not possible in WBANs mainly due to the reduced number of nodes and the particularly specialized tasks of each node.

On the other hand, Time Division Multiple Access (TDMA) is a good method to apply in WBANs due to its energy efficiency. Ameen et al. 12 proposed a MAC protocol for WBANs using out of band (on-demand) wakeup radio through a centralized and coordinated external wakeup mechanism. This protocol uses two types of wakeup scheduling: i) a wakeup scheduling for periodic traffic (normal data) and ii) a wakeup scheduling for random traffic (emergency data). In both normal and emergency traffic two radios are used (wakeup radio and main radio). The communication process takes place in two stages: in the first stage the wakeup radio is used to activate the node and, in the second stage, the main radio is used for control and data packet exchange. In this protocol, the coordinator maintains a table with the wakeup schedule of every node in the network. This table is constructed based on the traffic for every particular node while the wakeup intervals are calculated by the packet inter-arrival time. The authors proved through simulations that their method outperforms other MAC protocols in terms of energy efficiency and delay.

Although the MAC protocol proposed in [12] has been designed to save energy in WBANs, it lacks mechanisms to support energy harvesting capabilities. Besides, the protocol depends on a fixed, predetermined schedule which has two side effects: i) the state changes of the nodes from active to inactive mode could cause idle slots in the system, and ii) the flexibility is restricted, since it is cumbersome to add/remove nodes in the network.

The study of Boulis and Tselishchev [11] on MAC design for WBANs indicates that polling-based channel access offers significant energy gains compared to contention-based access. Regarding the latency (endto-end delay), the combination of short contention periods with long polling periods provides the most stable performance with respect to packet transmissions.

In this paper, taking into account the latest developments in WBANs, we propose a hybrid polling MAC protocol, so called HEH-BMAC, to address the effects of the human energy harvesting. Our contribution is summarized in the following:

1. To our knowledge, HEH-BMAC is the first MAC protocol designed to adapt to different energy conditions introduced by the human energy harvesting sources in the WBANs. In particular:

- The proposed protocol offers service differentiation by combining two different access methods: reserved polling access (ID-polling) for nodes of high priority and probabilistic random access (PC) for nodes of normal priority. 
- The ID/PC periods are dynamically adjusted according to the energy levels of the wireless nodes.

- Our protocol facilitates the network's flexibility by allowing the dynamic addition/removal of wireless sensor nodes.

2. We evaluate the performance of HEH-BMAC for different numbers of nodes in order to study the scalability of the protocol, while we elaborate on the network parameter tuning, i.e., transmission data rate and energy harvesting rate, to achieve the best possible performance for our protocol.

The rest of the paper is organized as follows. In Section 2, we describe our system model. In Section 3 , we introduce the HEH-BMAC design along with its frame structure and the protocol rules. In Section 4, we evaluate the performance of HEH-BMAC by extensive simulations. Finally, Section 5 concludes the paper.

\section{System model}

In our system model, we adopt a star topology, where the head (sink) is the Body Node Coordinator (BNC) responsible for setting up the network and collecting all the information transmitted by a number of lightweight and portable Body Nodes (BNs). The BNs have different functionalities and, consequently, different traffic load (i.e., packet inter-arrival time and packet payload).

The events detected by the BNs can be either signals carrying sensitive and vital information (e.g., electrocardiogram (ECG), electroencephalogram (EEG), etc.) or signals with random characteristics (e.g., motion, position, temperature, etc.). In order to model a most realistic scenario based on the above arguments, we adopt the same inter-arrival times $\left(\mathrm{IAT}_{\mathrm{BN}}\right)$ as in [26] for the event generation.

The energy collected by the harvester in the human body depends on factors such as the location of the node, as well as the harvester characteristics and dimensions. For this reason, choosing the best source/harvester for each sensor is also an important task in this type of networks. In HEH-WBAN, each sensor is connected to an energy harvester. We assume that the BNC has an external power supply and higher processing capabilities than BNs, while the BNs have a constant energy harvesting rate $\mathrm{K}_{\mathrm{EH}}$. The energy harvester must be able to harness the energy available at all times and for all states of the node (i.e., sleep state, idle state, transmission state $\left(T_{X}\right)$, reception state $\left(R_{X}\right)$ and dead state), as shown in Figure 2 thus maximizing the collection of

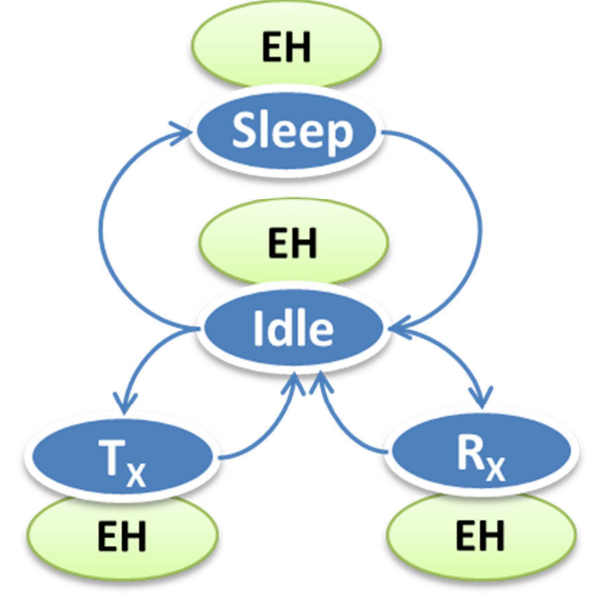

Fig. 2 BNs state transition diagram with energy harvesting.

available energy. Hence, the performance of the energy harvester directly affects the operation of the node, but not vice versa.

\section{Proposed Hybrid Polling MAC protocol Operated by Human Energy Harvesting (HEH-BMAC)}

HEH-MAC protocol has two operation modes: i) contention free ID-polling, and ii) probabilistic contention (PC) channel access. Hence, our protocol offers two levels of priority depending on the BN type. The high priority $\mathrm{BNs}$ are granted ID-polling access, while the normal priority BNs gain channel access with the probabilistic contention. In the following subsections we describe in detail the operation and the different modes of our protocol.

\subsection{ID-polling access mode}

In ID-polling, the BNC assigns a monitoring interval $\left(\mathrm{MIT}_{\mathrm{ID}-\mathrm{BN}}\right)$ to each node in this mode (ID-BN). Such monitoring intervals are stored and updated in a dynamic table. The MIT ${ }_{\text {ID-BN }}$ for each node is calculated using its respective $\mathrm{IAT}_{\mathrm{BN}}$ and $\mathrm{K}_{\mathrm{EH}}$ information. In this way, the BNC can anticipate the energy level of each sensor node and determine the polling periods based on a predictable schedule.

The BNC can apply an offset to the initial value of $\mathrm{MIT}_{\mathrm{ID}-\mathrm{BN}}$ (advance or delay) for each BN, to prevent that its $\mathrm{IAT}_{\mathrm{BN}}$ coincides with other nodes in time (Figure 3). For the allocation of this offset it is also taken into account the $\mathrm{IAT}_{\mathrm{BN}}$ and the $\mathrm{K}_{\mathrm{EH}}$, in order 


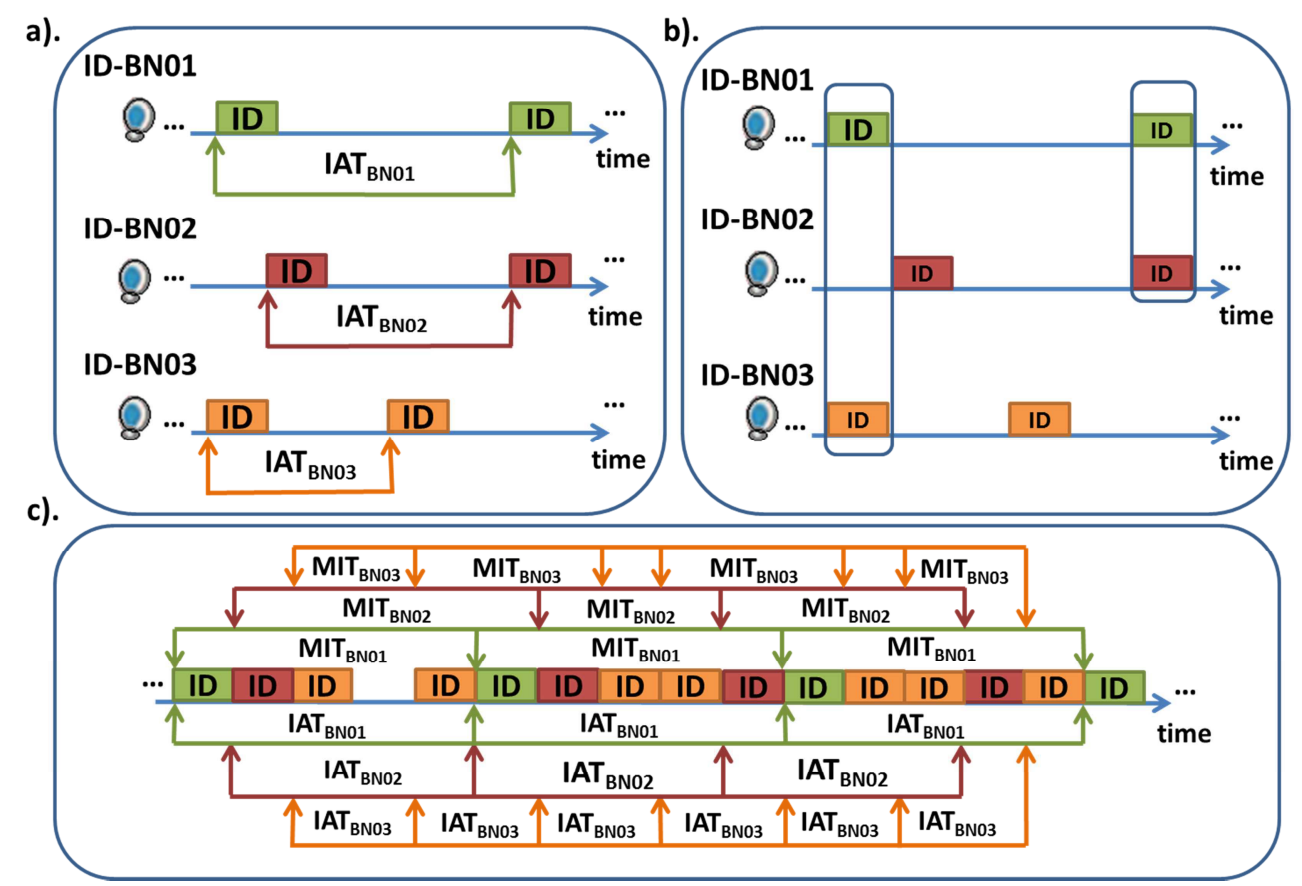

Fig. 3 Example of ID-polling access mode (a) $\operatorname{IAT}_{\mathrm{BN}}$ of the ID BNs (b) Overlap of the IAT $_{\mathrm{BN}}$ of the ID-BNs (c) MIT ID-BN of the ID-BNs using the offset.

to ensure the quality and quantity of clinical information collected. The BNC maintains the present values of MIT $_{\text {ID-BN }}$ in the dynamic table, which is updated after every transmission.

All nodes in the HEH-WBAN are assigned a unique ID for data security, data control and medical application. Figure 4 illustrates the communication process in ID-polling mode which takes place in three steps: i) the BNC transmits a polling packet containing the ID of the $\mathrm{BN}$ to be polled, ii) the polled $\mathrm{BN}$ responds with a data packet transmission, and iii) the BNC sends an acknowledgment (ACK) packet that confirms the successful reception of the data packet. As shown in Figure $4 \mathrm{~b}$, the ID-BN remains into the sleep state until its turn to transmit. Upon its turn, it wakes up and goes into the reception $\left(\mathrm{R}_{\mathrm{X}}\right)$ state to receive the ID-polling from the BNC. Once the communication is completed, the ID-BN turns again its radio to the sleep mode until the next round of polling.

\subsection{Probabilistic contention (PC) access mode}

The PC-access mode deals effectively with contention, achieving high throughput and maintaining fairness for single-hop networks. Besides, this mode offers the advantage of adapting to the changes in the energy harvesting rates, node failures or additions/removals of nodes.
In PC-access 17] 18, instead of ID-polling, the $\mathrm{BNC}$ broadcasts a control packet (CP-packet) that includes the value of the contention probability $(\mathrm{CP})$. When a $\mathrm{PC}-\mathrm{BN}$ (node in $\mathrm{PC}$-access mode) receives the CP-packet, it generates a random number $X_{i}$, where $X_{i} \in[0,1]$ and $i$ is an integer identifier of the node. If the value of $X_{i}$ is less than $\mathrm{CP}$, then the PC-BN transmits its data packet (see Figure 5 a); otherwise, the node transits to the idle state, waiting for the next CP-packet (see Figure 5b).

It is worth noting that CP is dynamically adjusted at the BNC according to an updating algorithm that takes into account the network load (in terms of traffic load and addition/removals of nodes) and the energy harvesting rate $\mathrm{K}_{\mathrm{EH}}$. The value of $\mathrm{CP}$ is updated in two cases: i) if no $\mathrm{PC}-\mathrm{BN}$ responds to the $\mathrm{CP}$-packet, the $\mathrm{BNC}$ increases the value of the $\mathrm{CP}$ threshold to increase the transmission probability of the PC-BNs, and ii) when there is a collision between two or more PC$\mathrm{BNs}$, the BNC decreases the value of the threshold to reduce the probability of collision. In case of successful transmissions, the current value of the threshold is maintained in the next CP-packet.

Some techniques that can be used to increase or decrease the value of the CP-packet are 18: additiveincrease multiplicative-decrease (AIMD), multiplicativeincrease multiplicative-decrease (MIMD), additive-increase additive-decrease (AIAD) and multiplicative-increase additive- 
a).
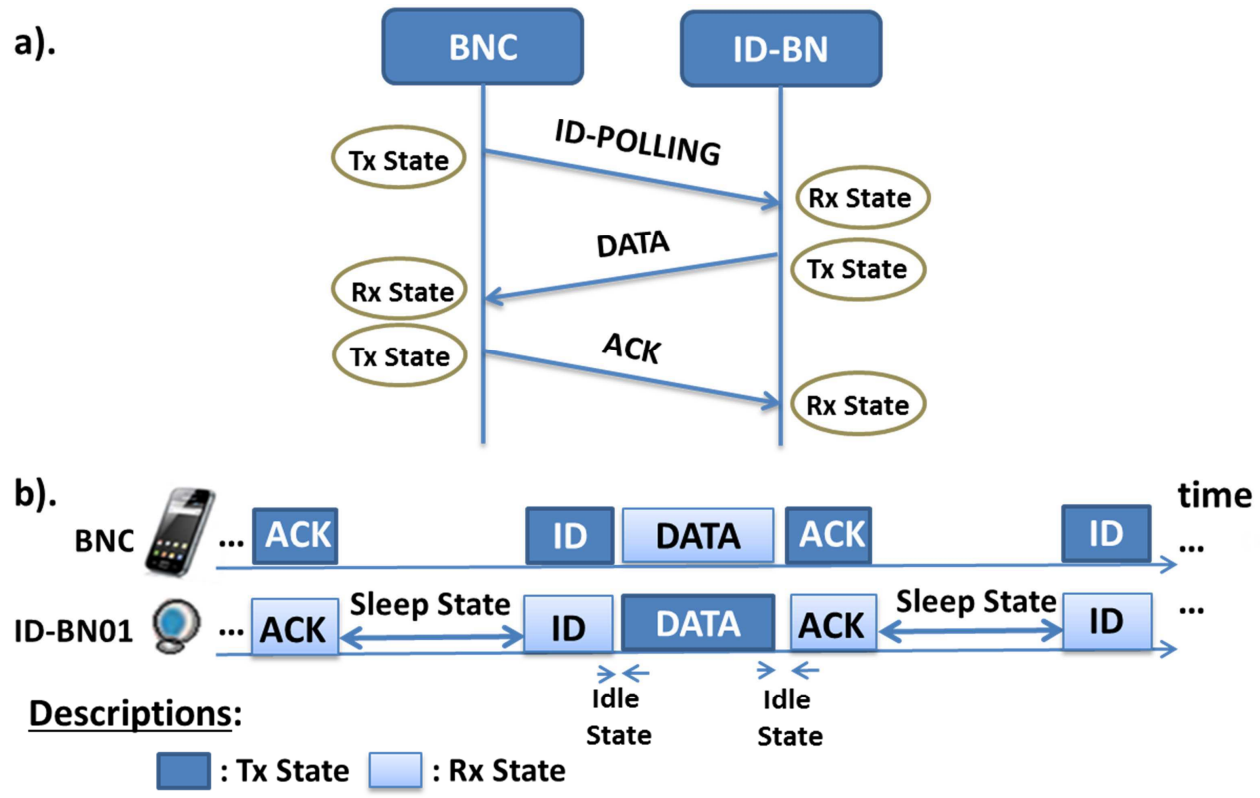

Fig. 4 ID-polling access mode (a) Data communication process (b) ID-BN states and transmission.

decrease (MIAD). In our model we use the AIMD technique because it provides higher throughput than the other schemes for single-hop scenarios 17 18. The AIMD [18] is a mechanism to increase the CP gradually by an increase factor $\alpha_{\mathrm{IN}}\left(0<\alpha_{\mathrm{IN}}<1\right)$ when polling is unsuccessful because of idle slots (i.e., $\mathrm{CP}_{(t+1)}=$ $\left.\mathrm{CP}_{(t)}+\alpha_{\mathrm{IN}}\right)$, or to decrease the $\mathrm{CP}$ by a larger decrease factor $\beta_{\mathrm{MD}}\left(0<\beta_{\mathrm{MD}}<1\right)$ in case of collisions in the network (i.e., $\mathrm{CP}_{(t+1)}=\mathrm{CP}_{(t)} \times \beta_{\mathrm{MD}}$ ).

An example is shown in Figure 5 . The BNC broadcasts a CP-packet containing the contention probability to define the threshold in that a PC-BN should transmit its data packet. In case of no packet reception, the BNC waits for a predefined time-out period ( $\left.\mathrm{T}_{\text {OUT }}\right)$, updates the CP-packet with the increased threshold and broadcasts the new value in the next PC round. The PC-BN transmits its data packet if $X_{i}<\mathrm{CP}$. If only one node transmits in the current $\mathrm{PC}$ round, the BNC sends the ACK packet to the polled PC-BN (successful transmission). In case of packet loss (unsuccessful transmission) due to collision between two or more PC-BNs, the BNC updates the CP-packet with the decreased threshold and the nodes are prepared to retransmit their data in the following PC round. All PC-BNs maintain a buffer to store the data to be retransmitted.

\subsection{HEH-BMAC (Hybrid ID-polling/PC-access)}

Our protocol combines both ID-polling and PC-access techniques to provide a better network performance. The HEH-BMAC is able to adapt to the network's changes with regard to the network size and the $\mathrm{K}_{\mathrm{EH}}$. In addition, the hybrid IDpolling/PC-access mode provides two types of priorities.

A BN can be classified as ID-BN or PC-BN according to the type of priority that has been assigned. The ID-BNs have fixed and collision-free medium access through ID-polling. In contrast, the PC-BNs have probabilistic contention-based medium access through PC-access.

The first task of our protocol is to assign the MIT ID-BN and calculate the duration of the data communication process $\left(\mathrm{DPC}_{\mathrm{ID}-\mathrm{BN}}\right)$ for each ID-BN. The $\mathrm{DPC}_{\mathrm{ID}-\mathrm{BN}}$ values and the current values of MIT $_{\text {ID-BN }}$ are stored in a dynamic table. This dynamic table is constantly updated with the next values of $\mathrm{MIT}_{\mathrm{ID}-\mathrm{BN}}$. The BNC manages the ID-polling process using the dynamic table. The use of this tool allows an ID-polling scheme based on a predictable schedule.

The second task performed by our protocol is to calculate the interval between two adjacent ID-polling periods. The BNC performs the calculation of this interval using the data provided in the dynamic list. If the time between two consecutive ID-polling periods is sufficient for a successful data transmission of a PC$\mathrm{BN}\left(\mathrm{T}_{\mathrm{PC}}\right)$, then this time is exploited for probabilistic contention ( $\mathrm{PC}$-access period). In case that time is not sufficient, the BNC remains idle waiting for the next IDpolling period. Figure 6 shows an example of ID-polling periods and PCaccess periods operating together. 


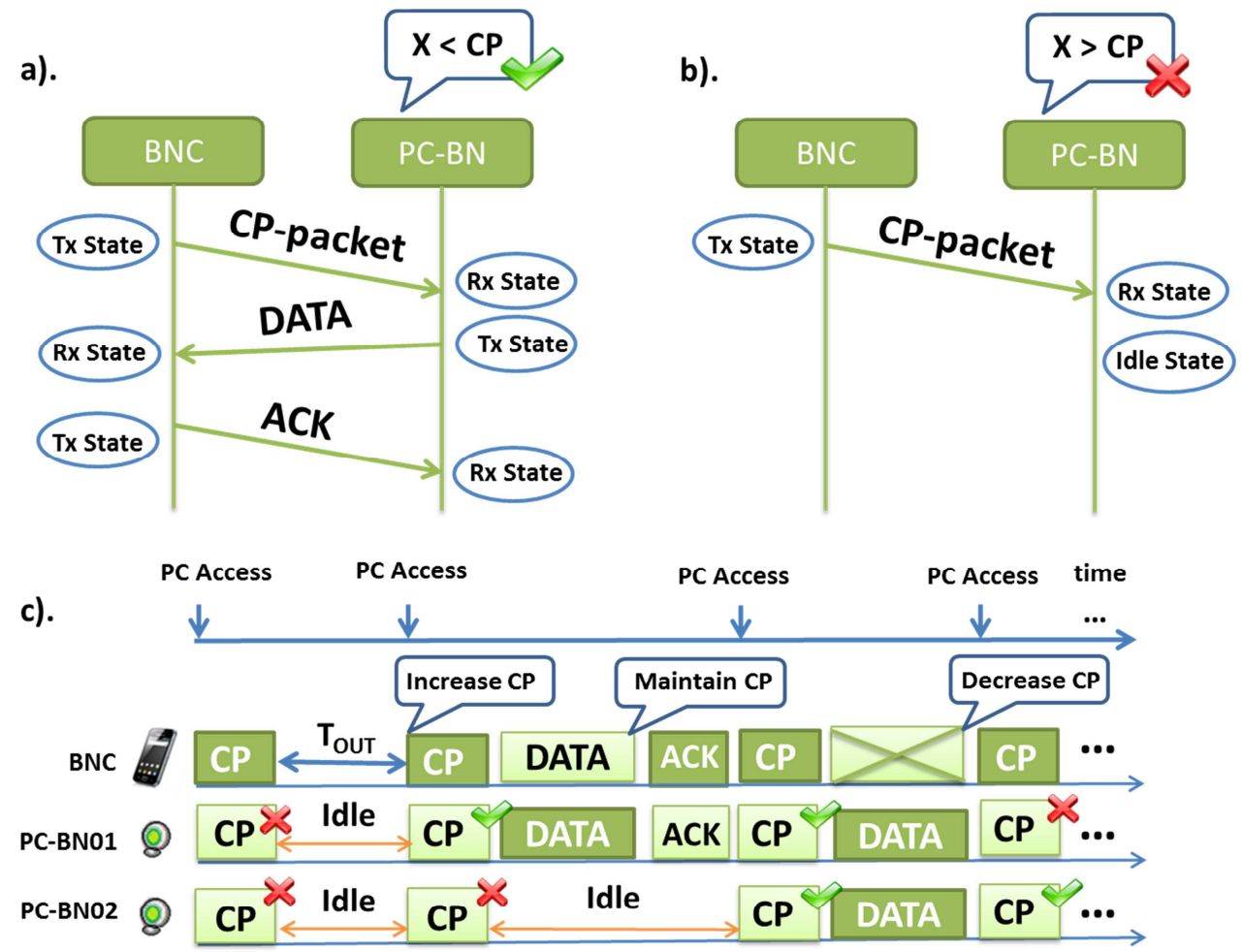

Descriptions:

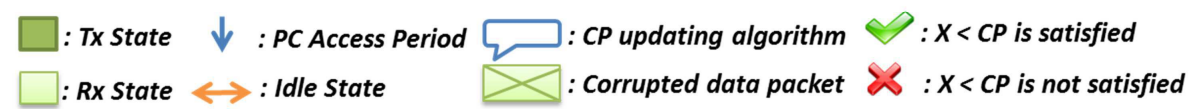

Fig. 5 PC-access mode (a) Data communication process when $X<\mathrm{CP}$ is satisfied (b) Data communication process when $X<\mathrm{CP}$ is not satisfied (c) CP updating algorithm and transmission process.

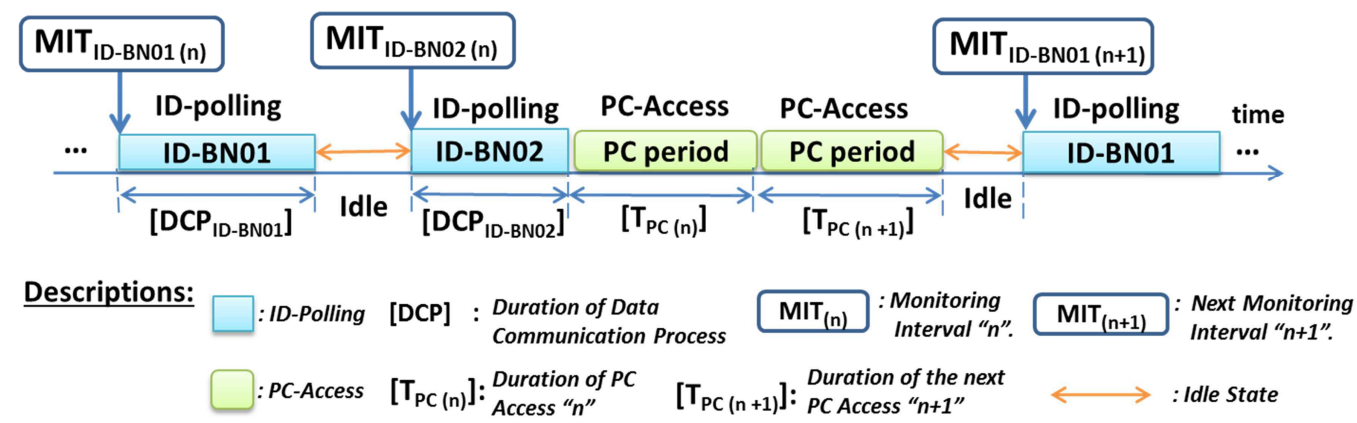

Fig. 6 ID-polling periods and PC-access periods in the Hybrid ID-Polling/PC-access.

Figure 7 shows an illustrative example of the HEHBMAC protocol running on a network with four nodes, where two nodes are in ID-polling mode and two nodes are in the PC-access mode. In detail, the protocol works as follows:

1. BNC performs the configuration and time calculations for the ID-BNs. BNC stores in a dynamic table the values $\mathrm{DPC}_{\mathrm{ID}-\mathrm{BN}}$ and current values of

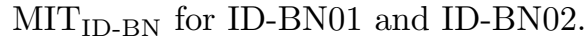

2. At instant $T_{1}$, BNC starts ID-polling access for IDBN01. Once the communication process has been completed, BNC updates the dynamic table with the next value of $\mathrm{MIT}_{\mathrm{ID}-\mathrm{BN} 01}$. ID-BN01 turns into sleep mode until its next ID-polling period. ID-BN02 remains in sleep state waiting its ID-polling period. The PC-BNs remain in sleep state if they have not packets for transmission; otherwise they turn to idle state, waiting for the beginning of the next PCaccess period. 


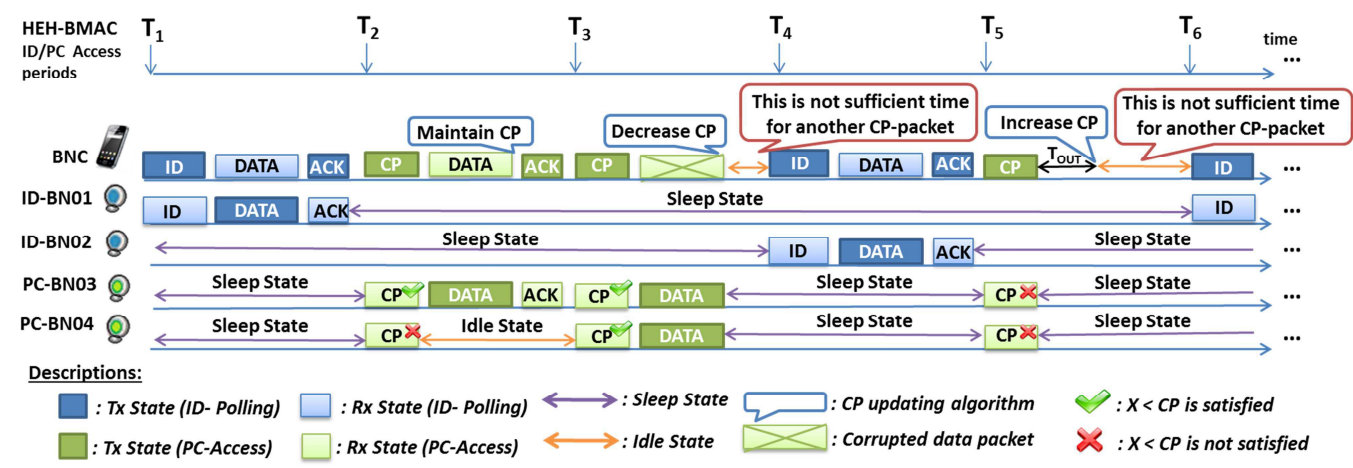

Fig. 7 Frame exchange in HEH-BMAC protocol.

3. BNC uses the dynamic list to calculate the interval between two adjacent ID-polling accesses $\left(T_{1}\right.$ and $T_{4}$ in this example). In this example, the interval is sufficient for two successful data transmissions in PC-access.

4. At instant $T_{2}, \mathrm{BNC}$ sends the CP-packet (starting $\mathrm{PC}$-access) to all $\mathrm{PCBNs}$ (i.e., $\mathrm{PC}-\mathrm{BN0} 03$ and PC-BN04). In this example, PC-BN03 randomly selects $X_{3}<\mathrm{CP}$ whereas PC-BN04 selects $X_{4}>\mathrm{CP}$. Hence, PC-BN03 gains access to the medium and starts its data transmission, whereas PCBN04 remains in idle state waiting the next $\mathrm{PC}$-access period. The $\mathrm{CP}$ updating algorithm maintains the current threshold value.

5. At instant $T_{3}, \mathrm{BNC}$ sends the next $\mathrm{CP}$-packet to all PC-BNs. In this example, the condition $X_{i}<\mathrm{CP}$ is satisfied for both PC-BN03 and PCBN04. Hence, both nodes transmit their data packets, resulting in a collision. According to the $\mathrm{CP}$ update algorithm, the BNC must decrease the CP threshold and include the updated value in the next CP-packet. In this example, however, the remaining interval (after the packet collision) is not sufficient for another PC-access. Therefore, the BNC remains idle until the next ID-polling period (which starts at $T_{4}$ in this example).

6. At instant $T_{4}$, the BNC starts ID-polling access for ID-BN02. Once the data transmission has been completed, the BNC updates the dynamic table with the next value of $\mathrm{MIT}_{\mathrm{ID}-\mathrm{BN} 02}$. In the meanwhile, ID-BN01 is in sleep state waiting its ID-polling period. The table is used to calculate the next interval between $T_{4}$ and $T_{6}$ and determine if there is enough time for PCaccess (in this example, the interval is sufficient for one successful data transmission in PCaccess).

7. At instant $T_{5}$, BNC broadcasts the CP-packet containing the new threshold value to all $\mathrm{PC}-\mathrm{BNs}$. In this example, neither PC-BN03 nor PC-BN04 se- lects a that satisfies the condition $X_{i}<\mathrm{CP}$. Hence, neither node transmits in the current PC-access.

8. The BNC waits for a predefined $\mathrm{T}_{\text {OUT }}$ and then increases the $\mathrm{CP}$ threshold value. In this case, since the remaining interval is not sufficient for another PC-access, the BNC remains idle until the next IDpolling period ( $T_{6}$ in this example).

\subsection{HEH-BMAC with energy harvesting}

As we have already mentioned, HEH-BMAC is energyaware, since it has been designed for energy harvesting. The behavior of each BN dynamically adapts to its energy level. The energy level of a node at a given moment can be defined as the energy stored in the battery plus the harvested energy minus the energy consumed by the radio interface. The modifications that energyawareness brings to our protocol are the following:

\section{ID-polling}

- Dynamic schedule: The BNC calculates the MIT $\mathrm{ID}_{\mathrm{BN}}$ using the information of $\mathrm{K}_{\mathrm{EH}}$ and $\mathrm{IAT}_{\mathrm{BN}}$ of each ID-BN. The MITID-BN has not a fixed value, since this time interval is continuously updated in the dynamic table, in order to know in advance the energy state of a node at any given time. In this way we can predict the responsiveness to an ID-polling for a given node, and take the decision whether to poll it or not.

- Polling-awareness: When a node receives an IDpoll packet, it checks its energy level. If the level is not sufficient the node does not respond to the poll but enters a sleep mode. In this case, the BNC assigns the time reserved for this IDpolling to the PC-access users.

\section{PC-access}

- Energy-awareness: The PC-BNs check both their energy level and their data packet buffers in order to decide whether to participate in the PCaccess. If their energy is below a certain level or 


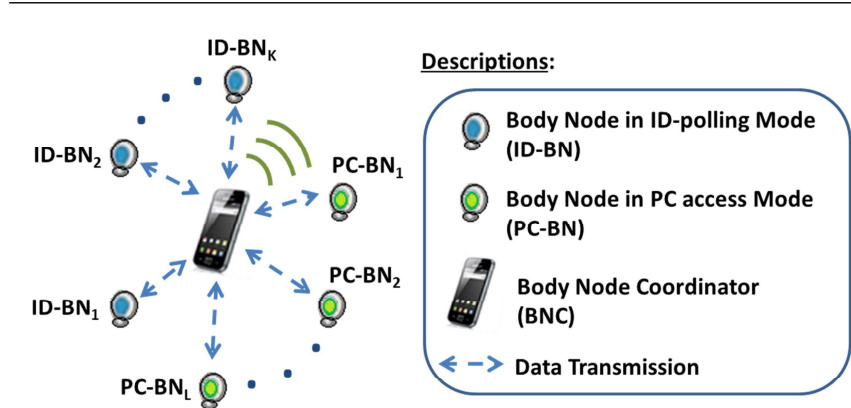

Fig. 8 Simulation scenario.

if their buffers are empty they enter into sleep mode. All PC-BNs will be in sleep state during the ID-polling.

- Polling-awareness: The PC-access mode is employed if there is enough time between successive ID-pollings. The BNC dynamically adjusts the $\mathrm{CP}$-packet according to the response of the $\mathrm{PC}-\mathrm{BNs}$ (through the CP updating algorithm).

\section{HEH-BMAC Performance Evaluation}

In order to analyze and evaluate the performance of HEH-BMAC, we have developed an event-driven MATLAB simulator that executes the rules of our protocol. In the following subsections we present the simulation setup along with the results of our experiments.

\subsection{Simulation Setup}

In our simulation model, we assume a star topology for a network consisting of a BNC, $K$ nodes in ID-polling mode and $L$ nodes in PC-access mode. The simulation scenario is depicted in Figure 8

The nodes in our simulations are typical medical sensors, whose traffic characteristics and priorities are shown in Figure 9. Let us recall that the high and normal priorities correspond to ID-polling and PC-access mode, respectively. The characteristics of the selected nodes in our experiments can be found in [26]. However, in the case of the ECG and Blood Pressure nodes, we adopt a slightly different aggregate traffic model which results in sample size of 120 bits and 96 bits, respectively. For this process, it was taken into account the bit rate and the delay requirements of healthcare data [25] [14].

The configuration parameters of the network have been selected according to the IEEE 802.15.6 PHYMAC specification [41] 37]. The simulation parameters are summarized in Figure 10.

\begin{tabular}{llccll}
\hline Physiological Signal & $\begin{array}{l}\mathbf{I A T}_{\text {BN }} \\
(\mathbf{m s})\end{array}$ & $\begin{array}{c}\text { Sample } \\
\text { Size } \\
\text { (bits) }\end{array}$ & $\begin{array}{c}\text { Data } \\
\text { rate } \\
\text { (kbps) }\end{array}$ & Priority & $\begin{array}{l}\text { Access } \\
\text { Method }\end{array}$ \\
\hline ECG signal & 20 & 120 & 6.0 & High & ID-Polling \\
Respiratory rate & 50 & 12 & 0.24 & High & ID-Polling \\
Blood Pressure & 80 & 96 & 1.2 & High & ID-Polling \\
Blood pH & 250 & 12 & 0.048 & Normal & PC-Access \\
Blood flow & 25 & 12 & 0.48 & Normal & PC-Access \\
\hline
\end{tabular}

Fig. $9 \mathrm{BNs}$ used in the simulation.

\begin{tabular}{|c|c|c|c|c|c|}
\hline Parameter & Value & Parameter & Value & Parameter & Value \\
\hline $\begin{array}{l}\text { MAC } \\
\text { Header }\end{array}$ & 56 bits & \multirow{4}{*}{ Data $\mathrm{T}_{\mathrm{x}}$ Rate } & $121.4 \mathrm{kbps}$ & $\mathrm{T}_{\mathrm{SIFS}}$ & $0.075 \mathrm{~ms}$ \\
\hline FCS & 16 bits & & $242.9 \mathrm{kbps}$ & $\mathrm{T}_{\text {OUT }}$ & $0.5 \mathrm{~ms}$ \\
\hline $\begin{array}{l}\text { PLCP } \\
\text { Preamble }\end{array}$ & 90 bits & & $485.7 \mathrm{kbps}$ & $\mathrm{P}_{\mathrm{TX}}$ & $27 \mathrm{~mW}$ \\
\hline $\begin{array}{l}\text { PLCP } \\
\text { Header }\end{array}$ & 31 bits & & $971.4 \mathrm{kbps}$ & $\mathrm{P}_{\mathrm{RX}}$ & $1.8 \mathrm{~mW}$ \\
\hline ACK & 72 bits & Control $\mathrm{T}_{\mathrm{x}}$ Rate & $121.4 \mathrm{kbps}$ & $P_{\text {SLEEP }}$ & $0.004 \mathrm{~mW}$ \\
\hline T-poll & 88 bits & PLCP $_{\mathrm{x}}$ Rate & $91.9 \mathrm{kbps}$ & $\mathrm{P}_{\text {IDLE }}$ & $0.712 \mathrm{~mW}$ \\
\hline
\end{tabular}

Fig. 10 System parameters.

We assume that the ID-BNs perform data transmission in real time (no packet retransmissions and no packets are stored in the buffer). In the PC-BNs retransmissions may take place when a collision occurs (packets are stored in the buffer). However, when the energy level of a node is very low (almost depleted), the node cannot proceed to the transmission or retransmission of packets and packet loss may occur. Moreover, the BNC maximum waiting time $\left(\mathrm{T}_{\mathrm{OUT}}\right)$ for a response from the nodes (ID-BNs or PC-BNs) is assumed to be equal to $0.5 \mathrm{~ms}$, while the Short Inter Frame Space period $\left(\mathrm{T}_{\mathrm{SIFS}}\right)$ is $0.075 \mathrm{~ms}$. For the AIMD CP updating algorithm, we use $\alpha_{\mathrm{IN}}=0.01$ and $\beta_{\mathrm{MD}}=0.5$, since these values give high throughput for single-hop scenarios [18.

Regarding the power supply, we assume that each node has incorporated an energy harvester that supplies power to a constant rate $\mathrm{K}_{\mathrm{EH}}$. In the beginning of our experiments, the nodes have empty batteries and, consequently, not sufficient energy for transmissions. We refer to this condition as the dead state, which is the default state when the node has not sufficient energy level. In the dead state, one node can only harvest energy (not consuming). Through the energy harvesting process, they collect energy in order to recover and start transmitting packets.

The energy consumed $\left(\mathrm{E}_{\mathrm{CON}}\right)$ in a given state (apart from the dead state) is determined by multiplying the power consumed $\left(\mathrm{P}_{\mathrm{STATE}}\right)$ with the time spent in this state $\left(\mathrm{T}_{\mathrm{STATE}}\right)$. On the other hand, the harvested energy $\left(\mathrm{E}_{\mathrm{EH}}\right)$ is calculated as the energy harvesting rate $\left(\mathrm{K}_{\mathrm{EH}}\right)$ multiplied by the energy harvesting time $\left(\mathrm{T}_{\mathrm{EH}}\right)$. 


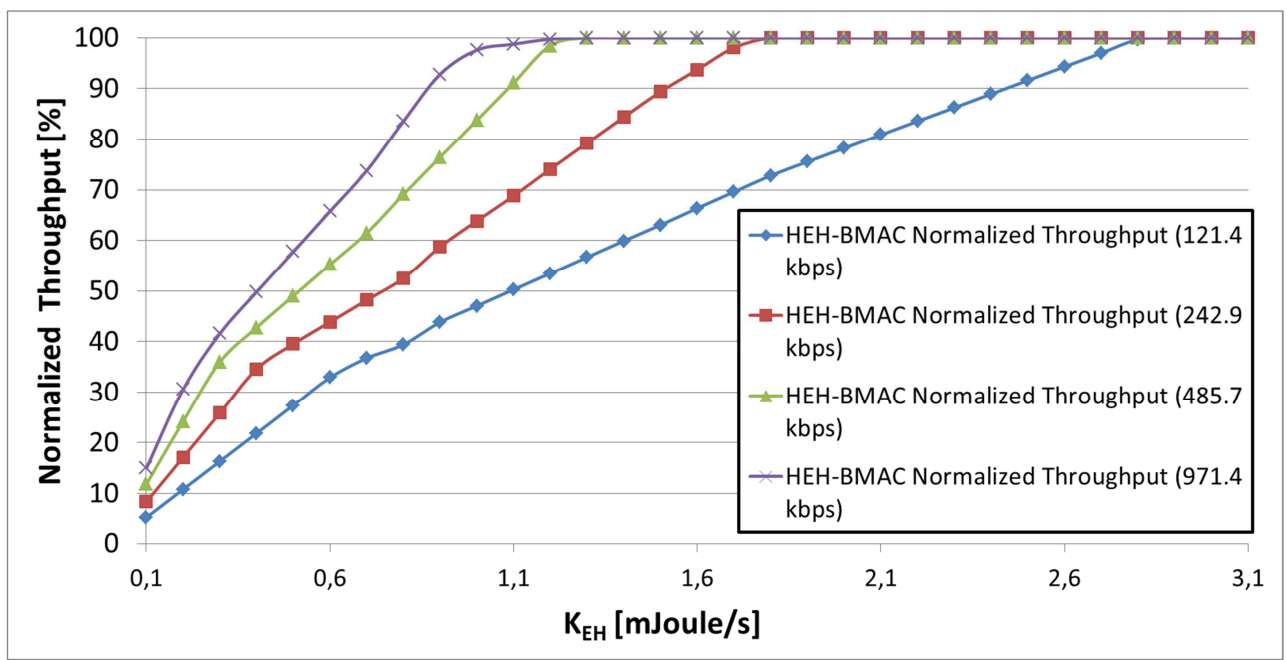

Fig. 11 Total normalized system throughput versus the energy harvesting rate, for the four data transmission rate $(K=3$, $L=2)$.

In order to evaluate the behavior of the HEH-BMAC protocol in energy harvesting conditions, we have conducted tests with different values of $\mathrm{K}_{\mathrm{EH}}$. The metric that is used to evaluate the performance of our protocol is the network throughput. Moreover, in order to evaluate the energy performance of our proposed protocol we use the energy efficiency metric [33.

\subsection{Performance results.}

Figure 11 presents the HEH-BMAC normalized throughput performance in the scenario described above versus the four IEEE 802.15.6 data transmission rates, for different values of $\mathrm{K}_{\mathrm{EH}}$. The normalized throughput of the network is defined as the percentage of data packets successfully transmitted divided by total amount of generated data packets. In this figure, we observe that for different data rates there is a specific value of $\mathrm{K}_{\mathrm{EH}}$ that achieves the maximum throughput. For example, in case of $485.7 \mathrm{kbps}$ and $971.4 \mathrm{kbps}$, the maximum throughput is reached for a harvester with a harvesting rate of $\mathrm{K}_{\mathrm{EH}}=1.2 \mathrm{mJoule} / \mathrm{s}$. However, for the same $\mathrm{K}_{\mathrm{EH}}$ but for transmission rates of $121.4 \mathrm{kbps}$ and 242.9 kbps we can achieve only up to $62.41 \%$ and $81.31 \%$, respectively. It is also worth noticing the similar behavior of $485.7 \mathrm{kbps}$ and $971.4 \mathrm{kbps}$ despite their great difference. This fact can be rationally explained if we consider that the protocol performance is dominated by the control transmission rate and the PLCP (Physical Layer Convergence Protocol) transmission rate. In this figure, we can also see the changes in the curve slopes which are a result from the interaction of two different channel access modes (ID-polling and PC-access).
Figure 12 shows the normalized throughput performance per node versus the $\mathrm{K}_{\mathrm{EH}}$, for the data transmission of $485.7 \mathrm{kbps}$. In this specific scenario, it can be observed that the nodes achieve improved performance in different threshold values of $\mathrm{K}_{\mathrm{EH}}$. As it can be seen for both ID-BNs and PC-BNs, a small/large IAT $_{\mathrm{BN}}$ (see Figure 9) value will require a longer/smaller $\mathrm{K}_{\mathrm{EH}}$ value in order to achieve the optimal performance.

Figure 13 presents the energy efficiency of a network composed of $K=3$ and $L=2$. The energy efficiency metric is defined as the total amount of useful data delivered over the total energy consumption. Energy efficiency increases when throughput is increased and when energy consumption is reduced. In this specific case, it can be seen that the energy efficiency is improving as the $\mathrm{K}_{\mathrm{EH}}$ increases. This is because as $\mathrm{K}_{\mathrm{EH}}$ increases, more energy is harvested in less time, allowing more data packets to be transmitted. The maximum energy efficiency achieved is $1.18 \mathrm{Mbits} / \mathrm{Joule}\left(\mathrm{K}_{\mathrm{EH}}=\right.$ $2.8 \mathrm{mJoule} / \mathrm{s}), 1.63 \mathrm{Mbits} / \mathrm{Joule}\left(\mathrm{K}_{\mathrm{EH}}=1.8 \mathrm{mJoule} / \mathrm{s}\right)$, $2.12 \mathrm{Mbits} / \mathrm{Joule}\left(\mathrm{K}_{\mathrm{EH}}=1.2 \mathrm{mJoule} / \mathrm{s}\right)$ and $2.34 \mathrm{Mbits} / \mathrm{Joule}$ ( $1.2 \mathrm{mJoule} / \mathrm{s}$ ) for data rates of $121.4 \mathrm{kbps}, 242.9 \mathrm{kbps}$, $485.7 \mathrm{kbps}$ and $971.4 \mathrm{kbps}$, respectively. To have a more complete picture for the energy performance, Figure 14 depicts the total energy harvested and the remained energy versus the energy harvesting rate, for the four data transmission rates. Both the collected and the remaining energy increase as the $\mathrm{K}_{\mathrm{EH}}$ increases. This occurs because the captured energy becomes greater than the energy consumed and energy remaining being stored in the energy storage of the nodes.

Figure 15 presents the normalized throughput performance of a network composed of $K=3 \mathrm{ID}-\mathrm{BNs}$ and $L=17$ PC-BNs. The packet inter-arrival time $\operatorname{IAT}_{\mathrm{BN}}$ 


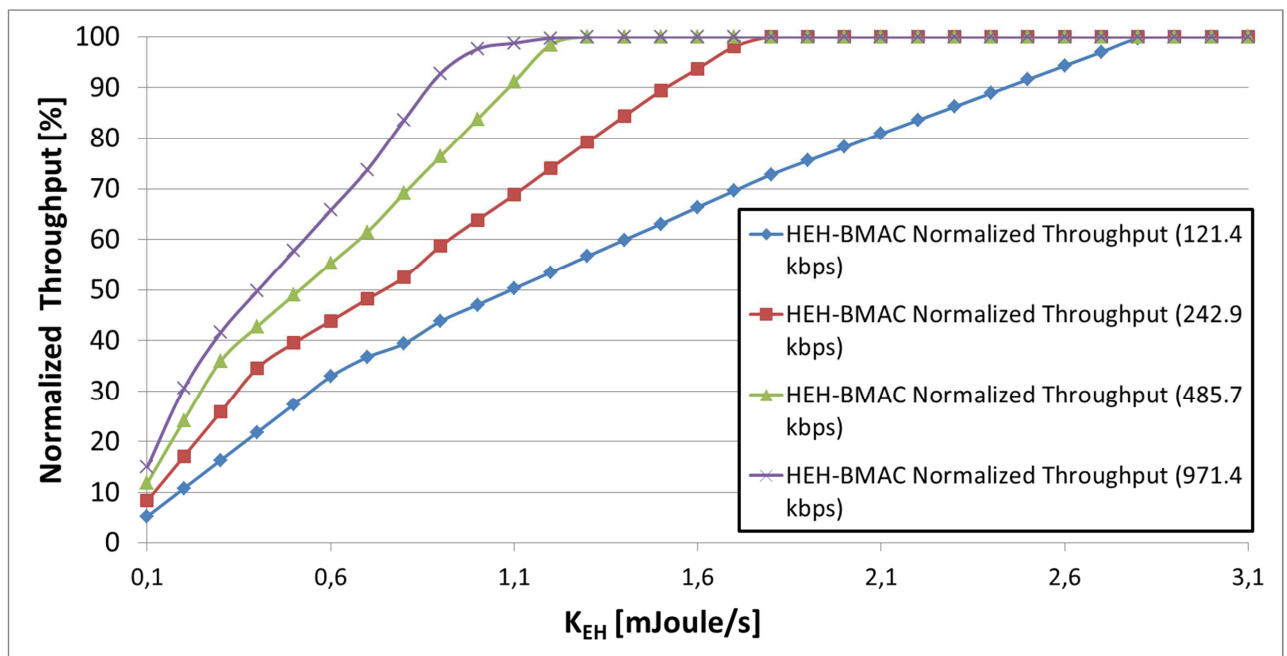

Fig. 12 Normalized throughput per node versus the energy harvesting rate (data rate $=485.7 \mathrm{kbps}, K=3, L=2$ ).

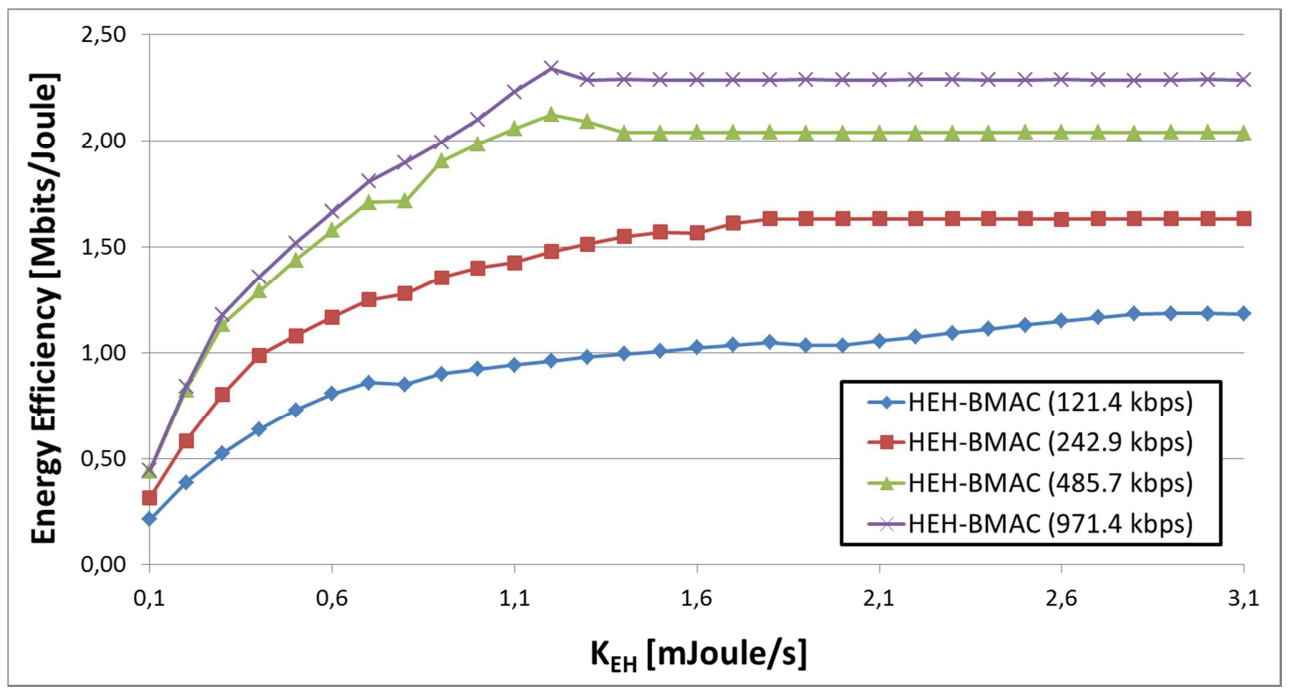

Fig. 13 Energy efficiency versus the energy harvesting rate, for the four data transmission rate $(K=3, L=2)$.

of ID-BNs is given by Figure 9, as before, however, different values of IAT $_{\mathrm{BN}}$ are applied to the PC-BNs. The throughput of the network is defined by the amount of data packets successfully transmitted within a certain period of time. If we increase the $\mathrm{IAT}_{\mathrm{BN}}$, fewer packets will be generated in a node, since the time interval between the generation of consecutive packets increases. In this specific case (data rate $=485.7 \mathrm{kbps}$, $K=1.3 \mathrm{mJoule} / \mathrm{s}$ ), it can be seen that the system performance is improving as the IAT $_{\mathrm{BN}}$ is reaching the value of $250 \mathrm{~ms}$ (throughput $\approx 100 \%$ ), while after this value the performance remains almost stable. The increase of the $\mathrm{IAT}_{\mathrm{BN}}$ implies a decrease of the number of packets to be transmitted, hence reducing the energy consumption of the node.

Figure 16 shows the normalized system throughput versus the number of PCBNs (ranging from 2 to
10), using a fixed data rate of $485.7 \mathrm{kbps}, 3$ ID-BNs and $\mathrm{K}_{\mathrm{EH}}=1.3 \mathrm{mJoule} / \mathrm{s}$. As it can be observed, the $\mathrm{HEH}-\mathrm{BMAC}$ protocol can maintain an almost stable system performance, since the total system throughput is not significantly affected when the number of PCBNs increases. Similar results have been obtained by changing the number of ID-BNs, keeping constant the number of PC-BNs. This can be explained by the fact that the time intervals for the ID-Polling and the PCaccess are constantly updated through the dynamic list, and packet collisions of the PC-BNs are dynamically resolved through the CP updating algorithm. 


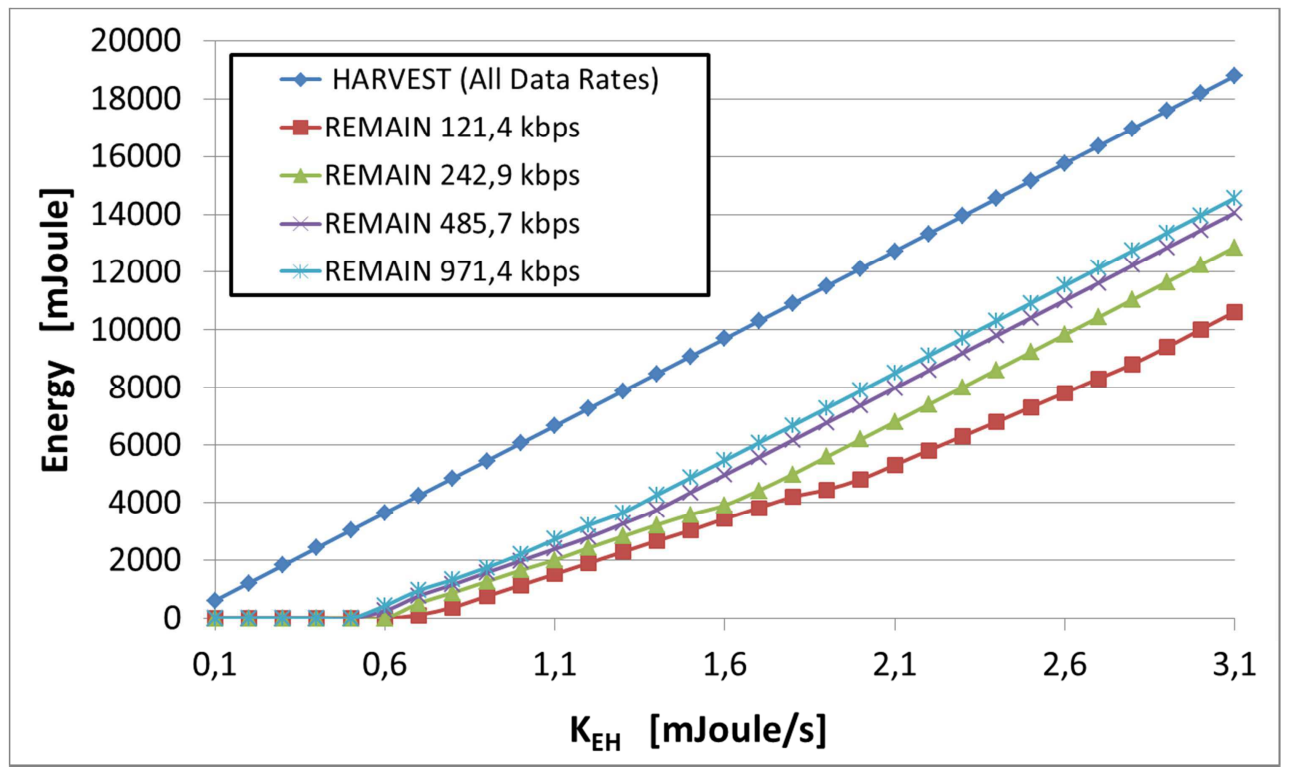

Fig. 14 Total system energy harvest and energy remain versus the energy harvesting rate, for the four data transmission rate $(K=3, L=2)$.

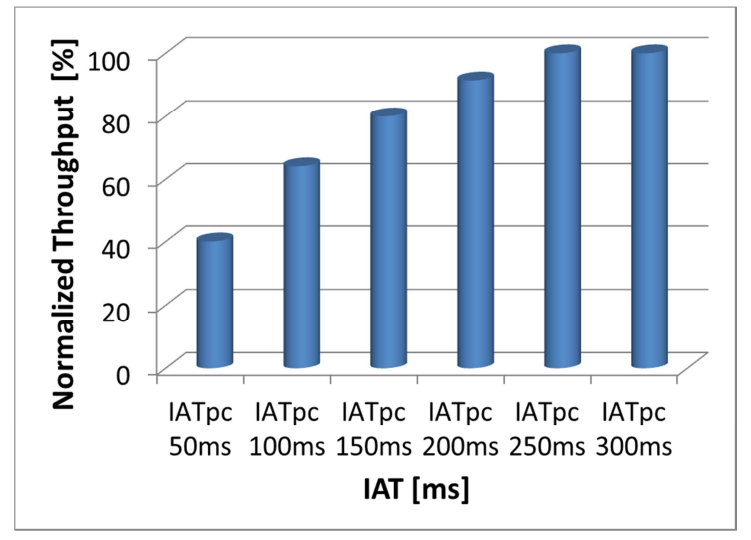

Fig. 15 Total normalized system throughput versus IAT of PC-BNs (data rate $=485.7 \mathrm{kbps}, K=3, L=17, \mathrm{~K}_{\mathrm{EH}}=1.3$ mJoule/s).

\section{Conclusions}

In this paper, a novel hybrid polling MAC operated by human energy harvesting for WBANs (HEH-BMAC) has been presented. The protocol adopts two modes of operation in order to provide priority differentiation to the sensor nodes and flexibility to the network. Comparing the behavior of our protocol in different conditions of energy harvesting rates, packet inter-arrival times and network size, we observe that HEH-BMAC dynamically adapts its operation to potential changes of these parameters. Our future work includes, among others, the analytical performance evaluation of our protocol, comparison with other MAC schemes and design

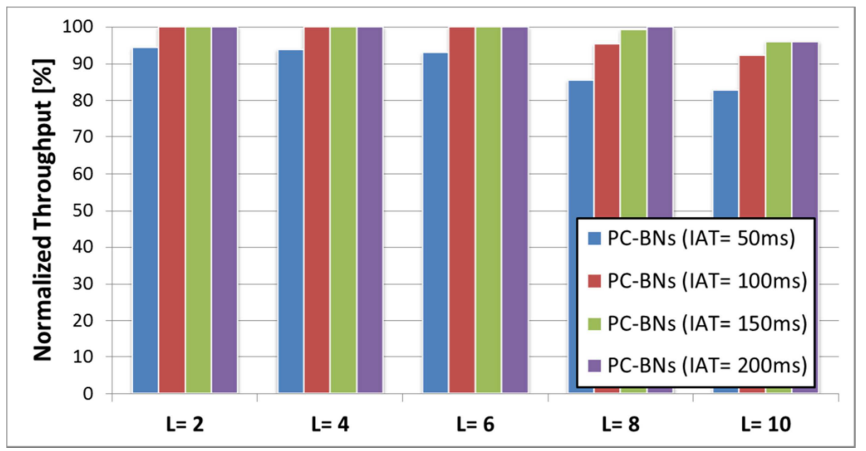

Fig. 16 Total normalized system throughput versus number of PC-BNs for different IAT (data rate $=485.7 \mathrm{kbps}, K=3$, $\mathrm{K}_{\mathrm{EH}}=1.3 \mathrm{mJoule} / \mathrm{s}$ ).

of smart algorithms to improve the energy efficiency, throughput and quality of service (QoS).

\section{Acknowledgements}

This work has been funded by the research projects CO2Green (TEC2010-20823), WSN4QoL (PIAP-GA2011-286047), ENIAC ARTEMOS (EUI2010-04252 and EUI2011-4349) and grant SENACYT-IFARHU (2702009-173).

\section{References}

1. Chen, X. and Xu, S. and Yao, N. and Shi, Y. . 1.6V Nanogenerator for Mechanical Energy Harvesting Using PZT Nanofibers. Nano letters, 10(6):2133-2137, May 2010. . 
2. He, Y. and Zhu, W. and Guan, L. Optimal Resource Allocation for Pervasive Health Monitoring Systems with Body Sensor Networks. Transactions On Mobile Computing, 10(11):1558- 157, 2011.

3. Latré, B. and Braem, B. and Moerman, I. and Blondia, C. and Demeester, P. . A survey on wireless body area networks. Wireless Networks, 17(1):1-18, 2011.

4. Li, H. and Chan, E. and Chen, G. AEETC-adaptive energy-efficient timing control in wireless networks with network coding. Telecommunication Systems, 45(4):289301, 2010. .

5. Markys, C. State of art in human powering devices. In 1st Energy Harvesting Research Theme Workshop, volume 2010, pages 1-32, Nov 2010. http://ehnetwork.org/events/workshop1/slides1.pdf. Accessed 12 July 2012.

6. Otal, B. and Alonso, L. and Verikoukis, C. . Highly reliable energy saving MAC for wireless body sensor networks in healthcare systems. Selected Areas in Communications, 27(4):553-565, 2009.

7. Ozel, O. and Tutuncuoglu, K. and Yang, J. and Ulukus, S. and Yener, A. Transmission with energy harvesting nodes in fading wireless channels: optimal policies. Selected Areas in Communications, 29(8):1732-1743, 2011.

8. Seyedi, A. and Sikdar, B. . Energy Efficient Transmission Strategies for Body Sensor Networks with Energy Harvesting . IEEE Transactions on Wireless Communications , 58(7):2116-2126, 2010.

9. Vullers, R.J.M. and Schaijk, R.V. and Visser, H.J., Penders, J. and Hoof, C.V. . Energy Harvesting for Autonomous Wireless Sensor Networks . Solid-State Circuits , 2(2):29-38, 2010. .

10. Yildiz, F. . Potential Ambient Energy-Harvesting Sources and Techniques. Technology Studies, 35(1):4048, 2009.

11. Boulis A. and Y. Tselishchev. Contention vs. polling: A study in body area networks mac design. In 7th International Conference on Body Area Networks (BodyNets), volume 2010, pages 98-104, Sep 2010.

12. Ameen, M.A. and Ullah, N. and Chowdhury, M. and Islam, S. and Kwak, K. A power efficient MAC protocol for wireless body area networks. EURASIP Journal on Wireless Communications and Networking, 2012(33):1-17, Feb 2012.

13. S. Balpande, S. Lande, and Rungta S. Modeling of pzt based power harvester and power control approach for pervasive node bsn. In IEEE International Advance Computing Conference (IACC), volume 2009, pages 327-332, Mar 2009.

14. Chen, B. and Pompili, D. Transmission of Patient Vital Signs Using Wireless Body Area Networks. Journal of Mobile Networks and Application. Mobile Networks and Application, 16(6):663-682, Dec 2011.

15. Dargie, W. and Chao, X. and Denko, M. . Modeling the energy cost of a fully operational wireless sensor network. Telecommunication Systems, 44(1-2):3-15, Jun 2011.

16. Drude, S. Requirements and application scenarios for body area networks. In 16th Mobile and Wireless Communications Summit IST, volume 2007, pages 1-5, Jul 2007.

17. Eu, Z.A. and Tan, H.-P. . Probabilistic polling for multihop energy harvesting wireless sensor networks. In IEEE International Conference on Communications (ICC), volume 2012, pages 271-275, Jun 2012.

18. Eu, Z.A. and Tan, H.-P. and Seah, W. K. G. Design and performance analysis of MAC schemes for Wireless Sensor Networks Powered by Ambient Energy Harvesting. Ad Hoc Networks, 9(3):300-323, 2011.
19. Fang, G. and Dutkiewicz, E. Energy efficient tdma-based mac protocol for wireless body area networks. In 9th International Symposium on Communications and Information Technology (ISCIT), volume 2009, pages 1455-1459, Sep 2009.

20. Garcia, M. and Sendra, S. and Lloret, J. and Canovas, A. . Hybrid nanogenerator for concurrently harvesting biomechanical and biochemical energy. American Chemical Society, 4(7):3647-3652, 2010.

21. Garcia, M. and Sendra, S. and Lloret, J. and Canovas, A. . Saving energy and improving communications using cooperative group-based Wireless Sensor Networks. Telecommunication Systems, 2011.

22. Gopalan, S.A. and Park J.-T. . Energy-efficient mac protocols for wireless body area networks: Survey. In International Congress on Ultra Modern Telecommunications and Control Systems (ICUMT), volume 2010, pages 739-744, Oct 2010 .

23. Gregori, M. and Payaro, M. Efficient data transmission for energy harvesting node with battery capacity constraint. In IEEE Global Communications Conference (GLOBECOM), volume 2011, pages 1-6, Dec 2011.

24. Hoang, D. and Tan, Y. . Thermal energy harvesting from human warmth for wireless body area network in medical healthcare system. In International Conference on Power Electronics and Drive Systems (PEDS), volume 2009, pages $1277-1282$, Nov 2009.

25. IEEE Std 11073-00101-2008. Health informatics PoC medical device communication, Part 00101: guide-guidelines for the use of RF wireless technology. ISO/IEEE, Dec 2008. 11073 ISO/IEEE Stds.

26. Khan, J. and Yuce, M. and Karami, F. . Performance evaluation of a wireless body area sensor network for remote patient monitoring. In 30th International Conference of the IEEE Engineering in Medicine and Biology Society (IEEE-EMBS), volume 2008, pages 1266-1269, Aug 2008.

27. Lim, J.C. and Bleakley, C.J. Trading sensing coverage for an extended network lifetime. Telecommunication Systems, 2011.

28. Lossec, M. and Multon, B. and Ahmed, H.B. and Goupil, C. Thermoelectric generator placed on the human body: system modeling and energy conversion improvements. European Physical Journal Applied Physics, 52(1):11103(1)-11103(10), 2010.

29. Marinkovic, S.J. and Popovici, E. and Spagnol, C. and Faul, S. and Marnane, W.P. . Energy-efficient low duty cycle MAC protocol for wireless body area network. Transactions on Information Technology in Biomedicine, 13(6):915-925, 2009.

30. J. Polastre, J. Hill, and D. Culler. Versatile low power media access for wireless sensor networks. In ACM Conference on Embedded Networked Sensor Systems (ACM SenSys), volume 2004, pages 95-107, Nov 2004.

31. Rapoport, B.I. and Kedsierski, J.T. and Sarpeeshkar, R. - A Glucose Fuel Cell for Implantable Brain-Machine Interfaces. Plos One, 7(6):1-15, 2012.

32. C. Renner, J. Jessen, and V. Tarau. Lifetime prediction for supercapacitor-powered wireless sensor nodes. In 8th GI/ITG KuVS Fachgespräch "Drahtlose Sensornetze" (FGSN'09), volume 2009, pages 55-58, Aug 2009.

33. Rodoplu, V. and Meng, T.H. Bits-per-joule capacity of energy-limited wireless networks. IEEE on Wireless Communications , 6(3):857-865, 2007.

34. W.K.G. Seah, Z.A. Eu, and H.-P. Tan. 1st international conference on wireless communication, vehicular technology, information theory and aerospace \& electronic systems technology (wireless vitae). In ACM Conference on 
Embedded Networked Sensor Systems (ACM SenSys), volume 2009, pages 1-5, May 2009.

35. A. Seyedi and B. Sikdar. Modeling and analysis of energy harvesting nodes in body sensor networks. In 5th International Workshop on Wearable and Implantable Body Sensor Networks (BSN), volume 2008, pages 175-178, Jun 2008.

36. F. Simjee and P.H. Chou. Everlast: long-life, supercapacitor-operated wireless sensor node. In International Symposium on Low Power Electronics and Design (ISLPED), volume 2006, pages 197-202, Oct 2006.

37. C. Tachtatzis, F. Di Franco, D. Tracey, Timmons N., and J. Morrison. An energy analysis of ieee 802.15.6 scheduled access modes. In IEEE Global Communications Conference (GLOBECOM), volume 2010, pages 1270-1275, Dec 2010.

38. N.F. Timmons and W.G. Scanlon. An adaptive energy efficient mac protocol for the medical body area networks. In 1st Wireless Communication, Vehicular Technology, Information Theory and Aerospace 83 Electronic Systems Technology (Wireless VITAE), volume 2009, pages 587-593, May 2009.

39. T. Van Dam and K. Langendoen. An adaptive energyefficient mac protocol for wireless sensor networks. In ACM Conference on Embedded Networked Sensor Systems (ACM SenSys), volume 2003, pages 171-180, Nov 2003.

40. J. Ventura and K. Chowdhury. Markov modeling of energy harvesting body sensor networks. In 21nd International Symposium on Personal, Indoor and Mobile Radio Communications (PIMRC), volume 2011, pages 21682172 , Sep 2011.

41. WBAN standard group (IEEE 802.15 Task Group 6). Standard for local and metropolitan area networks: Part 15.6: Wireless Body Area Networks. IEEE, Feb 2012. 802.15. 6-2012.

42. W. Ye, J. Heidemann, and D. Estrin. An energyefficient mac protocol for wireless sensor networks. In 21th IEEE International Conference on Computer Communications (INFOCOM), volume 2002, pages 1567-1576, Jun 2002 .

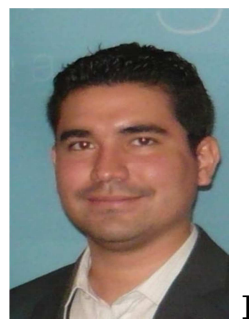

Ernesto Ibarra received the bachelor degree in biomedical engineering from the Universidad Latina de Panamá, Panamá, in 2007, the M.Sc. degree in biomedical engineering from Universitat de Barcelona and Universitat Politècnica de Catalunya, Spain in 2010. Currently, he is a PhD student in biomedicine at the Universitat de Barcelona. His areas of interest are: Energy Harvesting, Body Sensors, Body Area Networks, Radiation and Human Health. In the 2009 he was benefitted with a doctoral scholarship granted by SENACYT-IFARHU from the Panamanian Government.

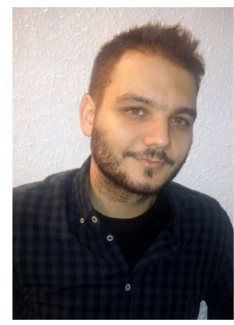

Angelos Antonopoulos received his MSc degree from the Information and Communication Systems Engineering Department (ICSDE) of the University of the Aegean in September 2007 and his Ph.D. degree from the Signal Theory and Communications (TSC) Department of the Universitat Politècnica de Catalunya (UPC) in December 2012. During the elaboration of his Ph.D., he has been involved in several national and European projects (ICARUS, CO2Green, Green-T, Greenet, etc). His main research interests include MAC protocols, RRM algorithms, network coding and energy efficient network planning, among others.

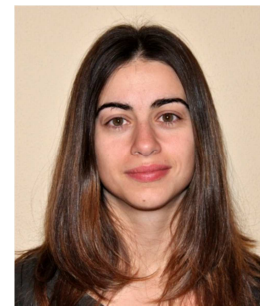

Elli Kartsakli received her $\mathrm{PhD}$

in Wireless Telecommunications from the Technical University of Catalonia (BarcelonaTECH) in February 2012. She holds a degree in Electrical and Computer Engineering from the National Technical University of Athens, Greece (2003) and an MSc in Mobile and Satellite Communications from the University of Surrey, UK (2004). Since 2009, she has participated in several national and European projects. Her research interests include wire- 
less networking, channel access protocols and energy efficient communication protocols.

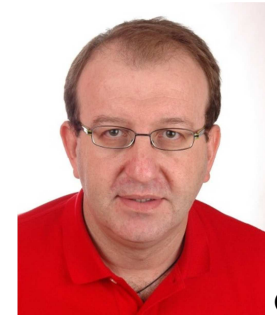

Christos Verikoukis received degree in Physics and M.Sc. in Telecommunications Engineering from the Aristotle University of Thessaloniki in 1994 and 1997, respectively. He got his PhD from the Technical University of Catalonia in 2000. Since February 2004 , he is a senior research associate in Telecommunications Technological Centre of Catalonia (CTTC). Before joining CTTC, he was research associate and projects coordinator in the Southeastern Europe Telecommunications \& Informatics Research Institute in Greece. He has been involved in several European (FP5 IST, FP6 IST \& Marie-Curie, FP7 ICT \& People, EUREKA) and national (in Spain and in Greece) research funded projects, while in some of them he has served at the Project or the Technical Manager. He has published over 100 journal and conference papers, 10 chapters in different books and 2 books. His research interests include MAC protocols, RRM algorithms, cross-layer techniques, cooperative and cognitive communications for wireless systems. 